\title{
Learned deconvolution using physics priors for structured light-sheet microscopy
}

\author{
Philip Wijesinghe $^{1}$, Stella Corsetti ${ }^{1}$, Darren J.X. Chow ${ }^{2,3,4}$, Shuzo Sakata ${ }^{5}$, Kylie R. Dunning ${ }^{2,3,4}$, and Kishan Dholakia ${ }^{1,6,7}$ \\ ${ }^{1}$ SUPA, School of Physics and Astronomy, University of St Andrews, North Haugh, St Andrews, Fife, KY16 9SS, UK \\ ${ }^{2}$ Robinson Research Institute, Adelaide Medical School, The University of Adelaide, Adelaide, SA, Australia \\ ${ }^{3}$ Australian Research Council Centre of Excellence for Nanoscale BioPhotonics, The University of Adelaide, Adelaide, SA, Australia \\ ${ }^{4}$ Institute for Photonics and Advanced Sensing, The University of Adelaide, Adelaide, SA, Australia \\ ${ }^{5}$ Strathclyde Institute of Pharmacy and Biomedical Sciences, University of Strathclyde, Glasgow, G4 ORE, UK \\ ${ }^{6}$ School of Biological Sciences, The University of Adelaide, Adelaide, South Australia, Australia \\ ${ }^{7}$ Department of Physics, College of Science, Yonsei University, Seoul 03722, South Korea
}

\begin{abstract}
Deconvolution is a challenging inverse problem in microscopy and underpins many computational imaging approaches, such as those that employ structured engineered point-spread functions. Here, we present a deep learning method that uses known physics priors to rapidly and robustly deconvolve and superresolve structured microscopy images. Specifically, we constrain a generative adversarial network with images generated with the known point-spread function of the system, and combine this with unpaired experimental data that preserves perceptual content. Our network overcomes a major barrier to deep learning adoption, namely, the need for 1,000-10,000s of paired training images. It is experimentally unsupervised and can be trained solely on a few hundred unpaired regions of interest. We demonstrate its performance on light-sheet microscopy with structured propagation-invariant Airy light fields, including in calibration beads, oocytes, preimplantation embryos, and excised brain tissue. Our network outperforms conventional deconvolution and achieves superior contrast, resolution and speed. This method aims to democratise the use of deconvolution with structured light fields, as it does not require data acquisition outwith the conventional imaging protocol.
\end{abstract}

deep learning | deconvolution | light-sheet microscopy | structured light | propagation-invariant light fields | Airy beam

Correspondence: pw64@st-andrews.ac.uk; kd1@st-andrews.ac.uk

\section{Introduction}

Deconvolution is a well-known problem in many fields of engineering and science (1-3). Specifically in computational imaging, the problem of deconvolution is one of the accurate reconstruction of the imaged object from data encoded by the imaging system. This encoding, for instance through the use of structured light fields and engineered point spread functions (PSF), can convey much greater information about the imaged object than is possible with conventional imaging (4). The use of structured light has enabled microscopy to breach the diffraction limit (5), to achieve axial sectioning using widefield detection (6) and to perform 3D localisation from axially varying PSFs (7). Propagation-invariant beam shapes, in particular, have extended the field of view and resolution $(8,9)$, and have demonstrated deeper penetration into scattering tissues $(10,11)$. These methods have enabled microscopy to push the envelope of many important trade-offs, such as those between the resolution, contrast, field of view, imaging speed and photo-damage.
A particular prominent area where structured light fields have enabled rapid, multiscale imaging at exceptional resolution is light-sheet microscopy $(\mathrm{LSM})(8,12)$. Propagationinvariant beams, such as the Airy and Bessel beams, have been a focus of many studies. These beam shapes can be generated using accessible optical components and implemented in simple optical geometries $(8,13,14)$. Despite their superior performance $(8,12)$, they are yet to be broadly adopted by the microscopy community due to the challenges of deconvolution. The barriers to broad adoption are two fold (3): first, conventional deconvolution methods suffer in poor signal-to-noise conditions, when the point-spread function (PSF) is structured and is not well known, and if scattering and speckle are present; and, second, deconvolution is time demanding and computationally intensive.

To overcome these major barriers, in this paper, we introduce deconvolution based on deep learning that is capable of robustly and rapidly deconvolving and super-resolving structured microscopy images. Recently, deep learning has emerged as a method to solve a variety of inverse problems in microscopy $(4,15)$. It has demonstrated the capacity to efficiently solve exceptionally ill-posed inversions and tackle non-linear noise (16). In microscopy, deep learning has enabled super-resolution from single images $(17,18)$, which may be considered as a form of deconvolution of blurring by the diffraction limit. Whilst achieving impressive results, a major hurdle to these data-driven approaches is that they require several 1,000 s to 10,000 s $(17,18)$ of experimentally acquired images, which additionally have to be co-registered to high-resolution experimental ground truths for supervised paired training. This often requires the user to have access to additional costly super-resolution imaging systems and hardware. Further, these methods learn, non-discriminately, the inversion of the physical system and the properties of the samples contained in the training set (4). This leads to poor generalisation and, thus, poor performance when a trained network is applied to samples outwith the training parameters (19). Thus, the user is required train bespoke networks for each system and sample imaged, which is a present and major barrier to broad adoption.

Instead of a data-driven approach, we develop a deep learning method that is informed by physics priors, i.e., a priori knowledge of the physics of the imaging system. The use 
of physics priors in deep learning have emerged to reduce the need for experimental training data and to direct training towards generalisation that is agnostic of the samples being imaged $(4,15)$. This can be achieved, for instance, by providing a network with an initial estimate of the image to be recovered (20), by controlling the spectral content (21), by incorporating simulations consistent with physics of diffraction and aberrations (22-24), or by tailoring training loss functions towards sparsity (25). In this paper, we follow the inspiration of such networks and make use of three key priors: (1) we make use of the known PSF of the imaging system to generate simulated paired data that is consistent with images that can be generated by the experiment; (2) the bases used to generate images are controlled in their spatial and spatialfrequency content, thus, provide control over the sparsity expected in the network outputs; and (3) we use experimental images to guide reconstruction towards the perceptual quality and power spectral content expected in biological samples.

Importantly, our method is entirely experimentally unsupervised, i.e., it does not require experimental ground truths, and may be trained rapidly on a single light-sheet volume, including the one that is desired to be deconvolved. Our method reduces the data burden from thousands of sub-pixel registered image pairs $(17,18)$, to a few hundred unpaired lowresolution image regions. We validate our method experimentally on LSM using an Airy beam in calibration beads, autofluorescent mouse oocytes and embryos, and excised mouse brain tissue. The method generalises well, learning primarily the inversion of the imaging operator, which we demonstrate by training only a single network for each beam shape, and applying it to all of the samples we subsequently image. We show that the deep learning method is superior to conventional deconvolution, and can be performed rapidly at $0.2 \mathrm{~s}$ per widefield image. Specifically, our method deconvolves images, demonstrating a more symmetric PSF, 98\% structured similarity index metric (vs. 85-91\% of conventional deconvolution), greatly improved image contrast and, importantly, super-resolution of a factor of $\sqrt{2}$ beyond the diffraction limit of the light field. Further, we illustrate that our method is 3-fold more resistant to model mismatch than conventional deconvolution, which is advantageous in the presence of misalignment or aberrations. Our approach to deep learning makes the use of structured light fields and deconvolution highly accessible as it does not require acquisition outwith the conventional imaging protocol. Towards this, we provide the deep learning code and data as open source to enable broad uptake of this approach among the imaging community.

\section{Results}

Network design. Figure 1 illustrates the architecture of our deep learning method under training within the context of the LSM setup. We perform deep learning based on a generative adversarial network (GAN) (26) and several tuned training loss functions (TLFs). The inverse problem, i.e., the transformation between an image encoded with a structured PSF (LR) to a high-resolution estimate of the ground truth, is learned using a Generator (G) network. Concurrently, another Discriminator (D) network is trained to identify network outputs (DL) and real ground truths (GT). During training, $G$ and $D$ play an adversarial min-max game, where $G$ seeks to fool D, and D seeks to accurately classify deep learning fakes (26). This adversarial training leads to the learning of strong image features that are consistent with the GT and that are difficult to capture using pixel-wise TLFs (27).

The network architecture is described in detail in the Methods Section, and briefly summarised here. The Generator is based on a 16-layer residual network (ResNet) (28), inspired by SRGAN (27) without upsampling. The Discriminator is inspired by a PatchGAN architecture (29), where local patches are classified independently to prioritise highfrequency image content.

Figure 1(a) illustrates the LSM geometry (see Methods for details). Structured illumination using an Airy light field is generated using a nematic spatial light modulator (SLM) in diffractive mode, encoding the appropriate cubic phase profile (the Fourier transform of the Airy function). The SLM enables precise control of the field intensity and phase in illumination. The desired pupil phase function (Fig. 1(c)), i.e., the spatial Fourier transform of the beam cross-section at focus, is projected onto the SLM (Supplementary Note 3). In this way, it is trivial to estimate the PSF of the LSM by combining the Fourier transform of the pupil, evaluated at the spatial coordinates established by the various lenses, and the diffraction-limited Gaussian spot size of the detection.

During a single training iteration, a batch of simulated images consistent with the LSM is fed into G. These images comprise two domains: a sparse collection of points, and speckle (Fig. 1(b)). Network inputs and their ground truths are formed, respectively, by convolving the images with the system PSF and a Gaussian spot set to $1 / \sqrt{2}$ of the diffraction limit of the objectives. The sparse points provide strong gradients for the network to learn the PSF shape. Speckle is a Fourier-transform pair to a uniformly distributed random phase and a Gaussian spectral amplitude. Thus, speckle images encode a wide spatial-frequency content with low sparsity. This pushes the network to prioritise feature learning (i.e., the transformation by the PSF) over content learning (i.e., the expectation of what microscopy images should look like), which benefits generalisation. During training, the network outputs are compared to the ground truths using the Discriminator (adversarial loss), and using L1-norm error (pixel loss). Concurrently, a random selection of experimental images are processed by G. The inputs and outputs of the network are compared using a pre-trained perceptual loss network (VGG-16 (30)), V (Fig. 1(b)), which focuses on the conservation of salient image content (31) (Supplementary Note 1). The addition of this TLF leads to the preservation of the power spectral density of experimental images, which is an important prior (32), and is effective at eliminating network artefacts. The combined loss of $\mathrm{G}$ is the linear combination of the adversarial, pixel and perceptual losses. The Discriminator is trained using conventional means (29), and training is alternated evenly between $\mathrm{G}$ and $\mathrm{D}$. 


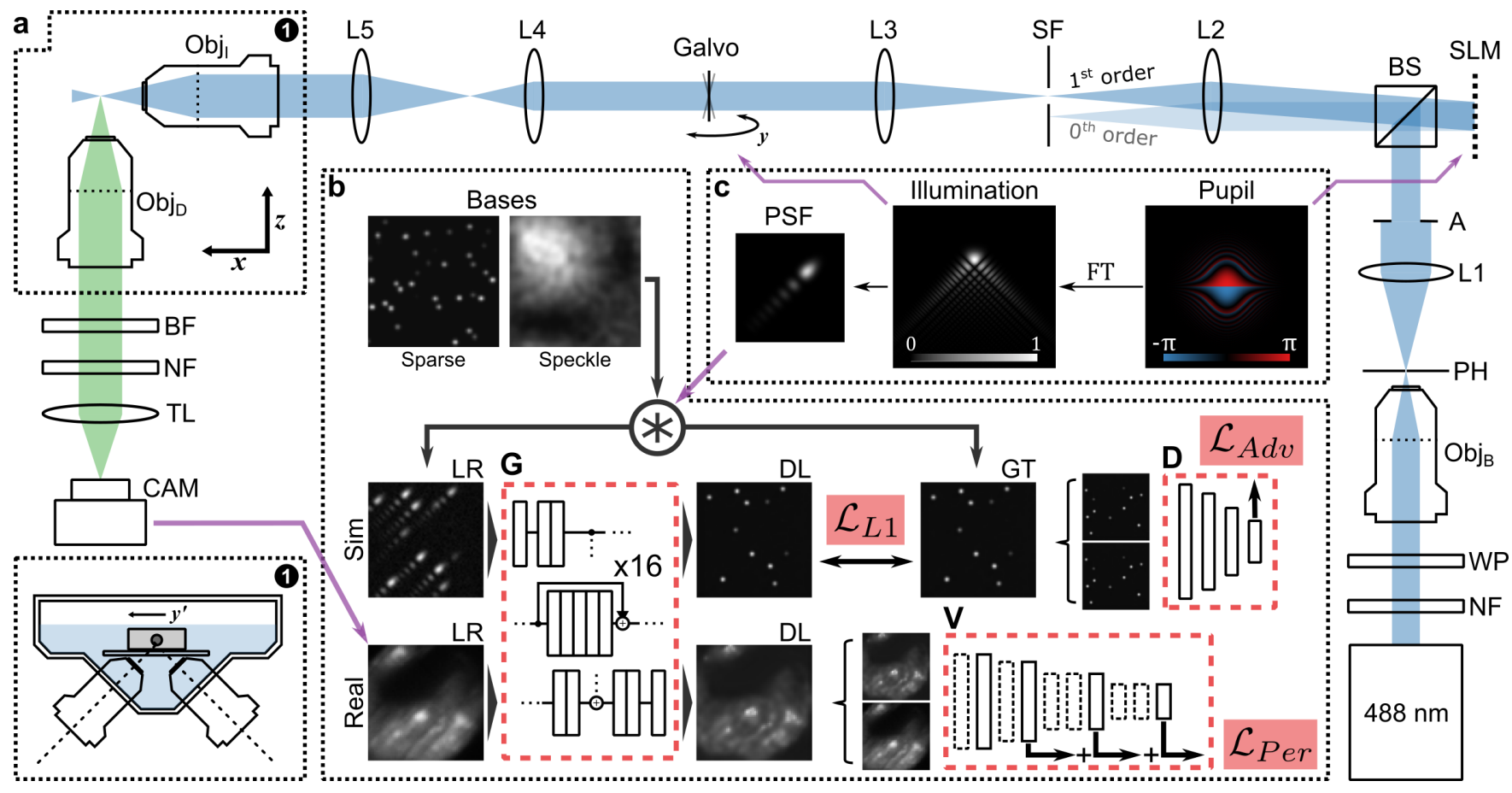

Fig. 1. Light sheet microscopy system and physics-informed learned deconvolution training. (a) Optical geometry of the system. Insets marked by (1) show the optical and physical positioning of the sample. (b) Deep learning method, consistent with (c) the physics of beam shaping using a spatial light modulator (SLM). Obj B, I, D: objectives for beam expansion, illumination and detection respectively. A $488 \mathrm{~nm}$ laser source is filtered using a notch filter (NF), half wave plate (WP), pinhole (PH) and aperture (A). L1-5: lenses. BS: beamsplitter. The SLM shapes the illumination field in diffraction mode to match the pupil function in (c), which is filtered using a spatial filter (SF) and scanned as a light sheet using a galvonometer driven mirror. Sample fluorescence filtered using a bandpass filter (BF) and a notch filter (NF), and imaged using a tube lens (TL) onto the camera (CAM). Deep learning learns from simulated images generated from sparse and speckle bases and the known PSF, and the real images captured by the CAM. During training, the generator $(G)$ learns from a combination of $L_{1}$ pixel loss, adversarial loss from the discriminator (D) and the perceptual loss from the perceptual loss network (V).

Our method and architecture results in a robust and generalised model. Importantly, we demonstrate this by training only a single network for each beam shape using only one experimental volume of mouse embryos ( 200 images). We apply this network, non-discriminately, to a wide array of samples, namely fluorescent beads samples, other embryos, oocytes, and turbid brain tissue.

Learned deconvolution. Our network accurately learns deconvolution with several advantages over conventional algorithms. For comparison, we use the well-known RichardsonLucy (RL) algorithm $(33,34)$. Firstly, we forego the common assumption that noise is a uniform and randomly distributed Gaussian process (3). Secondly, neural networks naturally use sparsity in images to improve reconstruction (35). Finally, as we will demonstrate, our network is less prescriptive than RL deconvolution and can tolerate greater model mismatch, for instance, when the theoretical and experimental PSFs differ. This means that our network will preserve its good performance even if the microscope is misaligned.

First, we quantify performance with solely simulated data on several representative beam types. Figure 2(a) compares the performance of our networks to RL deconvolution on images generated by propagation-invariant Airy and Bessel beams, and the conventional Gaussian beams. The generation of the various beam shapes is described in the Methods section. Briefly, the Airy beams are generated by a cubic phase in the pupil plane, with a scale-invariant $\alpha$ parameter that corresponds to the gradient of the cubic function. The Bessel beams are formed by a ring in the pupil plane, parameterised by a ring's radius and cross-sectional width. We later focus on the Airy and Gaussian beams in the experiment as they are readily generated by our LSM configuration.

From Figure 2(a) it is evident when using structured light fields, light-sheet images (LR) exhibit substantial side lobes. Here, the LR images include randomly distributed Gaussian (thermal) and Poisson (shot) noise. The conventional RL algorithm is able to reduce side lobes and present a spatially consistent image to the GT. However, RL-deconvolved images fail to present a visual quality matching that of the GT or the high-resolution Gaussian beam. Despite losing the structure, side lobes persist as elevated background intensities. We note that beam shapes marked by a black bar in Figure 2(a) carry similar spatial resolution. DL is capable of deconvolving LR images closer to the GT, eliminating the side lobes and presenting a Gaussian-like PSF. Figure 2(b) quantifies the performance of these methods using the structural similarity index metric (SSIM). It is evident that DL substantially outperforms RL deconvolution for all beam shapes. Specifically, DL achieves a 98\% SSIM, whilst RL ranges between $85 \%$ and $91 \%$. Further, we can see that performance is improved in both methods when the total size of the PSF is smaller.

$\mathrm{RL}$ deconvolution is an iterative technique that converges to the maximum likelihood solution based on Bayes' theorem when the noise is a linear Gaussian process (33). It is important to note that convergence of RL iterations leads to 


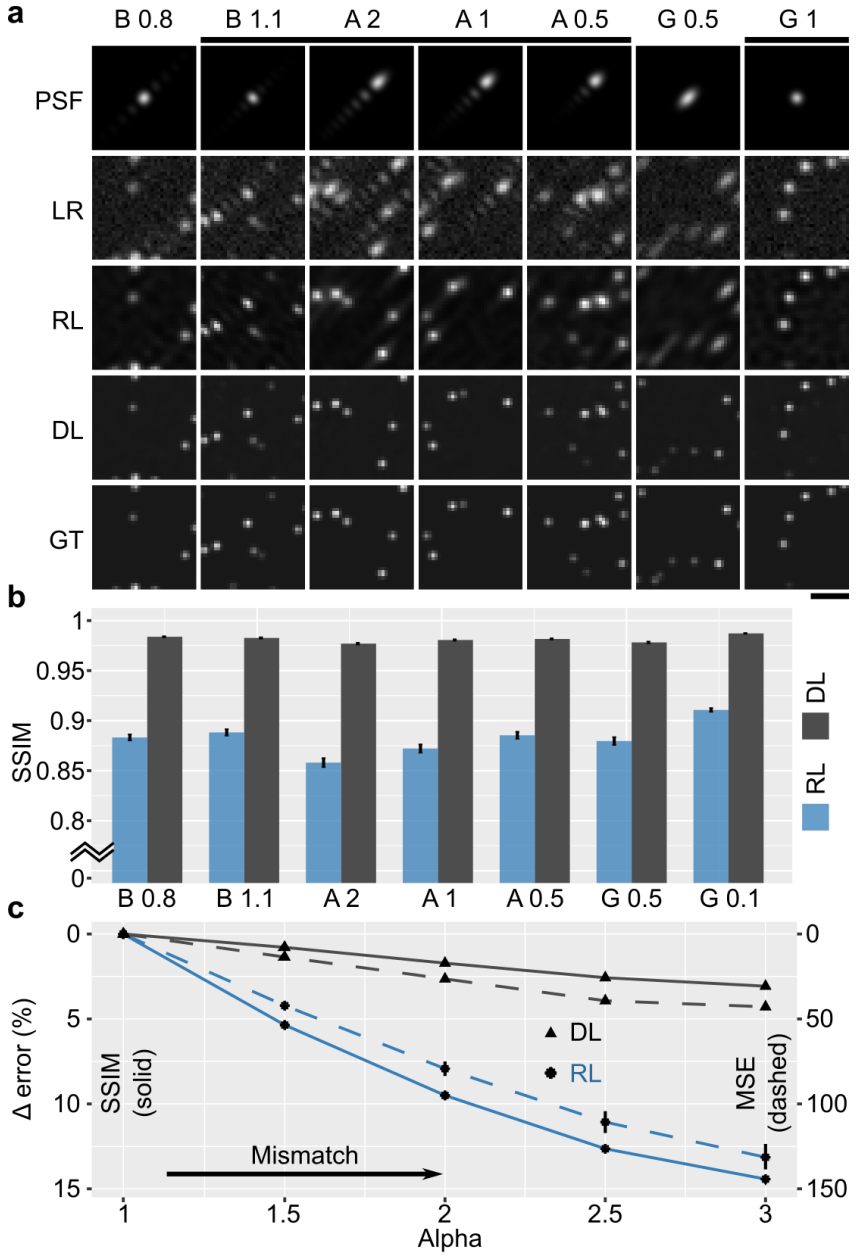

Fig. 2. Comparison of deep learning (DL) and Richardson-Lucy $(R L)$ deconvolution in simulated data. (a) Deconvolution of low-resolution noisy images (LR) simulated using beams (B: Bessel; A: Airy; G: Gaussian) visualised by their point-spread function (PSF) (parameters listed in Methods) using $R L$ and $D L$ algorithms and compared to the ground truth (GT). Scale bar is $20 \mu \mathrm{m}$. (b) Mean structured similarity index measure (SSIM) of RL and DL deconvolution for each beam shape evaluated in 512 simulated images (Error bar is standard error). (c) Relative change in error in deconvolution of an Airy beam due to model mismatch. $\mathrm{RL}$ and $\mathrm{DL}$ assume that the Airy $\alpha=1$; however, LR images were simulated with $\alpha$ in [1, 3]. Solid lines represent change in SSIM. The dashed lines represent the change in mean-squared error (MSE).

image artefacts and early truncation is often used as a spatial regulariser. Here, we truncated the RL iterations specifically to maximise the SSIM parameter for a best-case comparison.

A major challenge in deconvolution and in deep learning with priors is model mismatch (4). Here, this arises when the theoretical PSF differs from that of the imaging system, for instance, due to misalignment or aberrations. Figure 2(c) illustrates the robustness of our method to model mismatch. Specifically, we have generated LR images corresponding to Airy beams with $\alpha$ values in the range of 1 to 3 . However, to deconvolve the images, we have used a network trained on an Airy beam with an $\alpha=1$. Similarly, we have used a single Airy $(\alpha=1)$ PSF as a reference for the RL deconvolution. The matched training and testing case of $\alpha=1$ was used as a reference. Figure 2(c) shows the relative degradation in the SSIM and mean-squared error (MSE) error (to the known GT) as greater mismatch is introduced. It is clear that DL tol- erates much greater mismatch than RL deconvolution, on the order of 3-fold improvement to the error metrics. While blind deconvolution methods may overcome some issues of mismatch, we have observed that blind deconvolution struggles to converge when the PSF is structured (i.e., it is not monotonically decreasing from a central peak) and when images are not spatially sparse, which is consistent with previous observations (3). It is important to note that while we have visualised performance on sparse point-scatterers, quantitative data in Figure 2 comprises in equal part images of speckle.

Figure 3 extends the demonstration of our DL method to experimental data of $200-\mathrm{nm}$ beads. Using the SLM, we readily generate a Gaussian illumination (Fig. 3(a)), and compare it to the Airy beam with $\alpha$ parameters of $0.5,1$ and 2, respectively (Figs. 3(b-d)). The red bar indicates the Rayleigh range of each beam shape, which corresponds to the field of view available for LSM. The acquired LSM images are labelled as LR, and are deconvolved using DL and RL deconvolution. For each beam shape, the network was trained on one acquisition of tissue data (see Methods) and applied to all experimental data included in this work. The 200-nm beads are sub-diffraction limit, thus, represent the system response or the PSF. We can see that DL offers superior image quality to RL deconvolution. This is clear in the transverse profile of the beads marked by the red box. The Airy function can be seen in the LR profiles. The RL deconvolution removes the structure of the side lobes, but fails to substantially reduce the envelope, leading to an asymmetric PSF. The superior performance of DL is evident by the sharp and more Gaussian-like PSF.

In addition to decoding the structure, DL leads to a superresolution of individual beads beyond the diffraction limit of the light field. We can quantify this performance over many beads in the field of view using the autocorrelation function (Fig. 3(e)), which represents the shift invariance and relates to the depth or axial LSM resolution. From the Wiener-Khinchin theorem, we know that the ACF of intensity is a Fourier pair to the power spectral density, i.e., the square of the modulation transfer function (MTF). Figure 3(f) shows the MTF of the Gaussian LSM and the Airy $(\alpha=1)$ beams compared to their theoretical MTF, as well as the RL and DL deconvolutions. We can see that the MTF in the acquired images match theory well (dashed lines). The dotted line marks the $5 \%$ threshold of the MTF, which corresponds approximately to the full-width at half-maximum (FWHM) of the PSF. We note that the intersection of the Gaussian and Airy beam MTFs at the $5 \%$ threshold indicates their capacity to carry similar high-frequency content. However, this information is not well conveyed by the Airy images in the spatial coordinate space as illustrated by the widened ACF. As expected, RL deconvolution decodes Airy images (LR) to improve the ACF width with little benefit seen in the MTF. Remarkably, DL expands the MTF beyond that of the Gaussian beam, and extends the crossing of the $5 \%$ threshold by a factor of $\sqrt{2}$. This improved performance is also evident by the rapidly decreasing ACF. The FWHM resolution was quantified as $2.6 \mu \mathrm{m}$ in the Gaussian and Airy images, and $1.7 \mu \mathrm{m}$ 

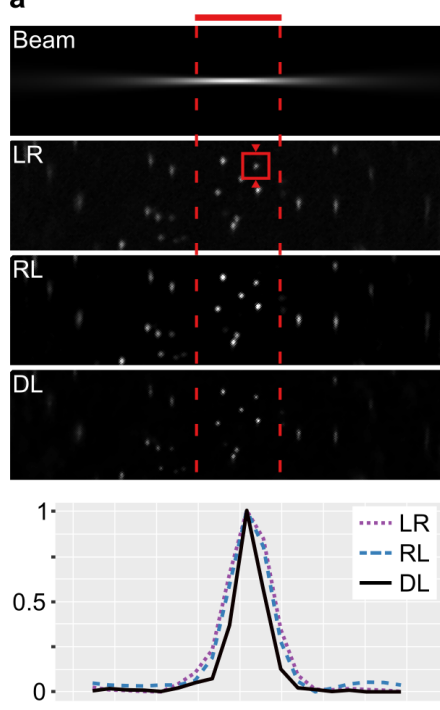

e

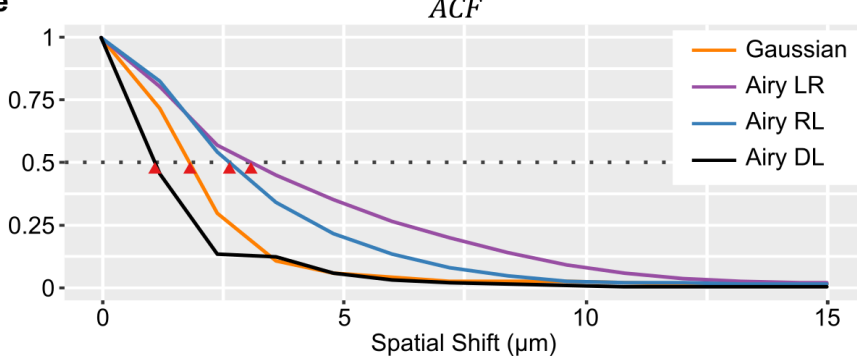

b
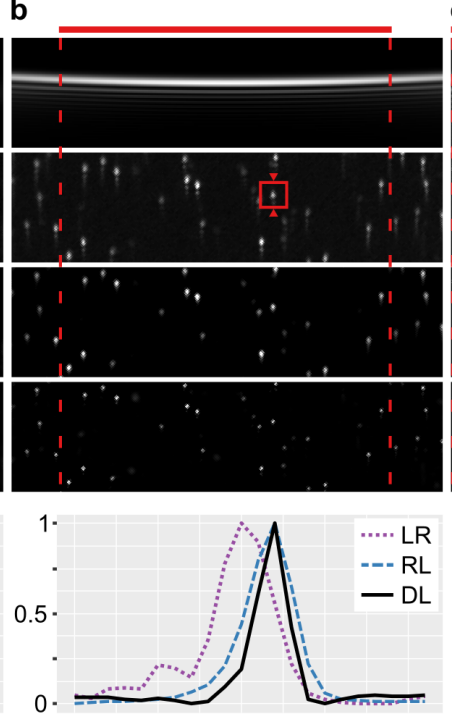

0 .

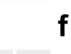

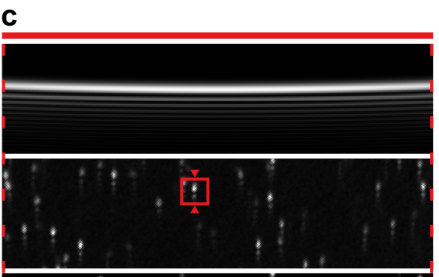

d
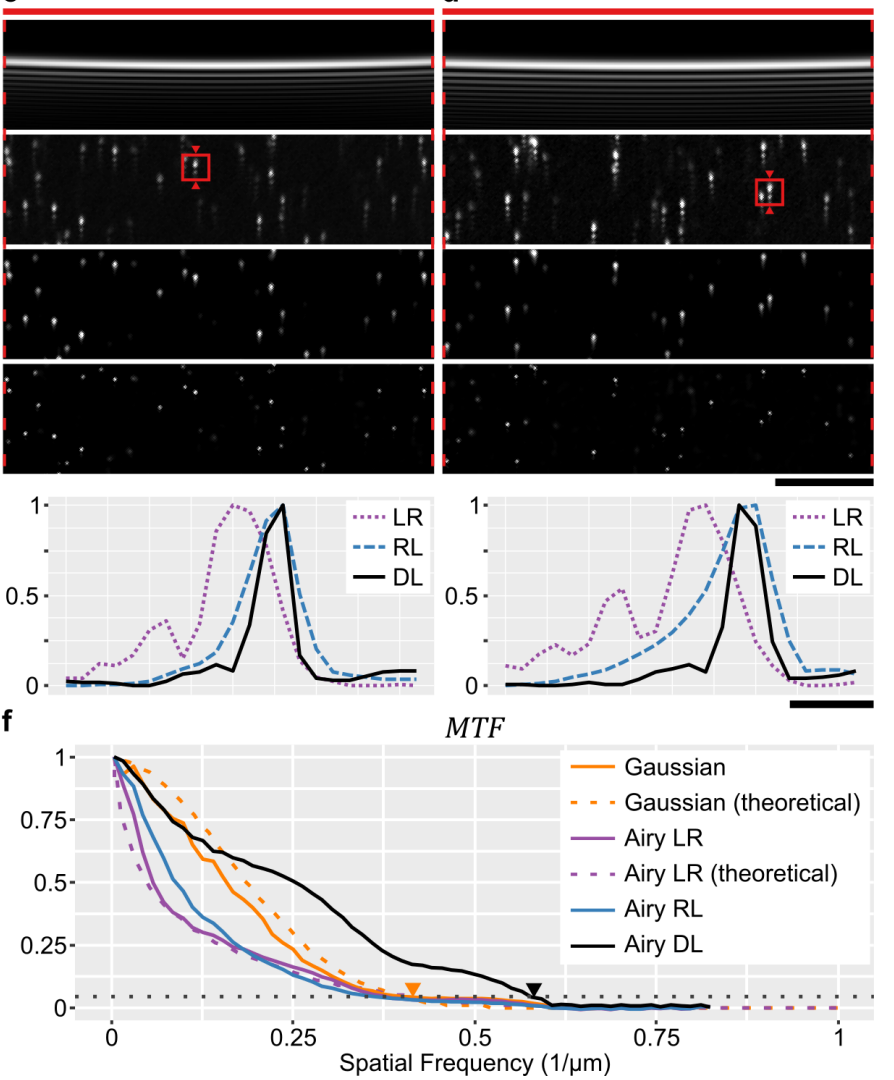

Fig. 3. Learned deconvolution in beads using a (a) Gaussian, (b) Airy ( $\alpha=0.5$ ), (c) Airy ( $\alpha=1$ ), and (d) Airy ( $\alpha=2)$ beam. (a-d) Show the illumination beam and the corresponding acquired LSM image (LR), which is deconvolved using Richardson-Lucy (RL) and deep learning (DL) methods. Profiles correspond to the bead marked by a red square. Red dashed lines mark the Rayleigh range of each beam. Scale bars are $50 \mu \mathrm{m}$ for images and $5 \mu \mathrm{m}$ for plots. (e) Autocorrelation function (ACF) and (f) Modulation transfer function (MTF) with respect to depth of the Gaussian and Airy ( $\alpha=1)$ LSM images.

with the use of DL. This exemplifies the power of deep learning as a method to solve inverse problems that leverages sparsity, learned noise statistics and the imaging operator to both decode information and extend the bandwidth limit.

Here, DL has provided the Airy LSM a $\sqrt{2}$ enhancement in resolution over the equivalent Gaussian beam at focus. At the same time, it preserved its substantially wider FOV of up to $372 \mu \mathrm{m}$ over the $27-\mu \mathrm{m}$ FOV of the Gaussian beam, a more than 13 fold improvement. The accessible and rapid inference of DL, the wide FOV and the ease of generating propagation-invariant beams confers exemplary potential to multiscale imaging applications. To demonstrate this, we explore our DL method in mouse oocytes and embryos, and in excised mouse brain tissue.

Oocytes and Embryos. Rapid, minimally invasive imaging of embryos is important for diagnostics and may aid in the monitoring of embryo development following in vitro fertilisation (IVF) $(36,37)$. LSM is particularly promising in this area due to its rapid volumetric imaging, reduced photodamage and low cost compared to confocal microscopy (36). Our DL-assisted Airy LSM is capable of performing cross-sectional imaging of the entire depth of an embryo $(\sim 180 \mu \mathrm{m})$ in one shot. Further, it is able to convey high-resolution morphological and functional information from autofluorescence.
Figure 4 demonstrates the performance of DL deconvolution in mouse blastocyst-stage embryos. The blastocyst is the final stage of preimplantation embryo development, and comprises two sub-populations of cells: the inner cell mass (ICM) which forms the fetus, and the trophectoderm (TE) which leads to the development of all extra embryonic tissues, including the placenta (38). Figure 4(a) shows a crosssection through a blastocyst imaged with an Airy $(\alpha=1)$ beam and deconvolved using RL and DL methods. The same region was imaged using a Gaussian beam (G). We can see that DL readily decodes the Airy PSF from the LR image, and matches and exceeds the image quality presented by the Gaussian LSM. We illustrate this further by taking a profile marked by the red triangles. In Figure 4(b), we can see that DL clearly distinguishes two autofluorescent features with a contrast that exceeds that in G. These features are not clear in the LR image and only minimally pronounced when using RL deconvolution.

We now focus on demonstrating the advantage of using the Airy beam LSM with deep learning deconvolution compared to the conventional Gaussian LSM, which represents the most popular embodiment of LSM. Figure 4(c) shows several cross-sections from different blastocyst-stage embryos imaged with a Gaussian LSM (G) compared to matching regions imaged with an Airy LSM with DL, hereafter referred to as 'Airy DL' for succinctness. Visually, we see an improve- 

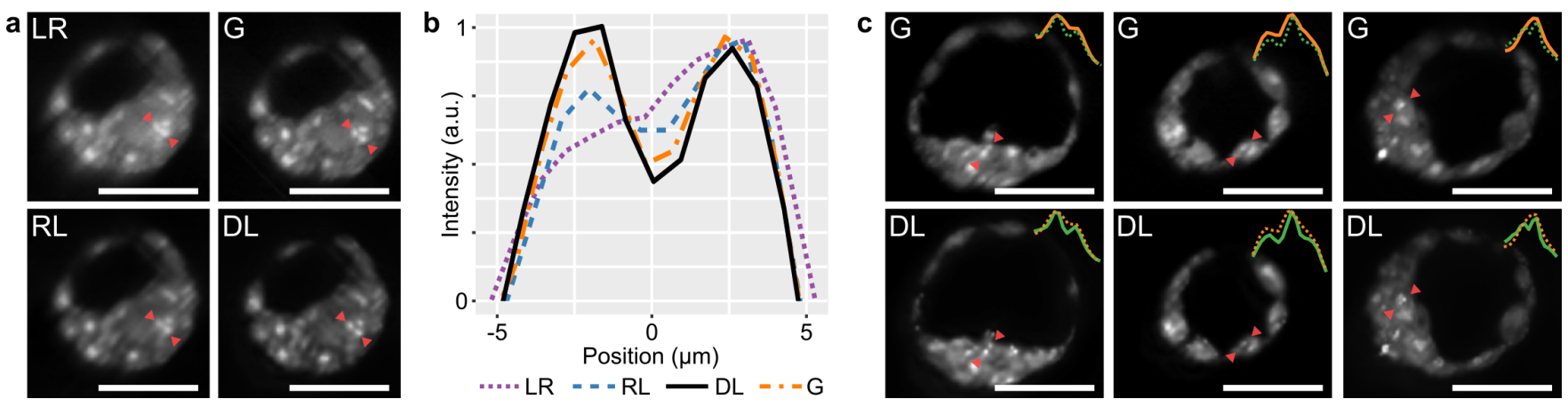

Fig. 4. Cross-sectional images of mouse blastocyst-stage embryos. (a) Airy LSM image (LR) is deconvolved with Richardson-Lucy (RL) and deep learning (DL) deconvolution, and compared with a Gaussian LSM (G). (b) Intensity profiles marked by the red arrows in (a). (c) Cross-sections of blastocyst-stage embryos imaged with a Gaussian LSM (G) and compared to an Airy LSM with deep learning deconvolution (DL). Profiles marked by the red triangles are displayed as insets. Scale bar is $50 \mu \mathrm{m}$.

ment in the contrast and resolution in the DL images. Line profiles marked by the red triangles and inset into the figures further illustrate that DL is able to distinguish autofluorescent features with a higher spatial resolution. We attribute these bright features to active mitochondria performing high levels of metabolic activity and, thus, generating higher amount of intracellular fluorophores, such as flavin adenine dinucleotide (FAD) (39). This is consistent with the excitation and emission filters in our LSM setup. Resolution, intensity and count of these features may be an important metric for IVF success (37). It is clear that the added super-resolution assists the counting of individual sharp features, and the FOV in depth enabled doing so in a single snapshot.

Figure 5 compares a Gaussian LSM to Airy LSM with DL in mouse cumulus oocyte complexes (COCs). COCs comprise of an oocyte at the centre, surrounded by much smaller cumulus cells. Imaging of the COC may provide an opportunity to assess the health of the oocyte prior to IVF (39). The larger field of view encompassing multiple COCs emphasises the major advantage of using an Airy beam, namely, the extended depth of field (DOF). Figure 5(a) and (b) show enface sections through several COCs acquired with a Gaussian and Airy DL LSM. The 1-mm transverse FOV illustrates the large volumetric imaging capacity of LSM. Cross-sections marked by red dashed lines are visualised in Figure 5(c) and (d). These sections are maximum intensity projections over a $50 \mu \mathrm{m}$ range to emphasise COC morphology. Figure 5(c) and (d) mark the focus and DOF of the illumination beams by the red triangle and bar, respectively. It is clear that much of the Gaussian cross-sections are out of focus. Airy DL is able to maintain the COC volume in the focal region and demonstrates clear morphological features and enhanced resolution. In the insets of Airy DL, we can see the clear demarcation of cumulus cells, even in regions well beyond the focus available to the Gaussian beam.

The zoomed-in insets in Figure 5(a) and (b) show the oocyte arrested at the metaphase II stage of meiosis. During metaphase, the chromosomes are aligned and held in place by the meiotic spindle along a plane termed the metaphase plate. This can be seen by the white line that separates two darker spherical regions that depict the spindle barrel. This morphology is particularly prominent in the Airy DL images, and can be confirmed for all oocytes by inspecting the volume (see Data Availability). This is an essential event for an oocyte undergoing nuclear maturation, necessary for fertilisation. As described for the blastocyst-stage embryo, the small bright regions within the oocyte are indicative of metabolic activity of mitochondria through FAD autofluorescence. The multitude of morphological features enhanced by Airy DL over a wide FOV and depth range underlines its potential for label-free and low-phototoxic imaging for embryo health and IVF success. Revealing the source and implications of these markers would be of considerable interest for future bioimaging studies.

Brain. LSM is a powerful emerging technique for neuroscience (40). LSM is well positioned to address multiscale imaging at depth, combining imaging at the resolutions and timescales of synapses, over tailored fields-of-view corresponding to whole brains of model organisms or regions of pathogenesis. However, penetration depth is a present challenge, especially in single-photon modalities. The Airy light field has several properties that are of great benefit in this area. Beyond its propagation invariance, Airy light fields are self-healing. If a portion of the field is blocked or scattered, the beam cross-section will reform into its expected Airy function. This has led to several observations that Airy light fields penetrates on the order of $30 \%$ deeper into tissues $(10,41)$.

Furthermore, we demonstrate our technique in excised mouse brain that expresses enhanced YFP in parvalbuminpositive $(\mathrm{PV}+)$ neurons. Figure 6(a) and (b) show enface sections with depth taken from cortical areas, including the hippocampus. These regions were selected from a larger acquired volume, indicated in the cross-sectional image (Fig. 6(c)). To the right of each image are zoomed-in regions corresponding to the areas marked by the white dashed rectangles. Because $\mathrm{PV}+$ neurons provide somatic inhibition in the hippocampus (42) and the neocortex (43), we hypothesise that axonal boutons in perisomatic regions (around the cell body) clearly mark PV+ neurons in Airy DL images as bright rings (red asterisks). While PV+ neurons are clearly seen, axonal terminals (bright small spots) around the cell bodies (large dark circles) are also visible in other neurons in lower number. Close to the surface, the enhancement in contrast and resolution of the Airy DL, for instance when in- 

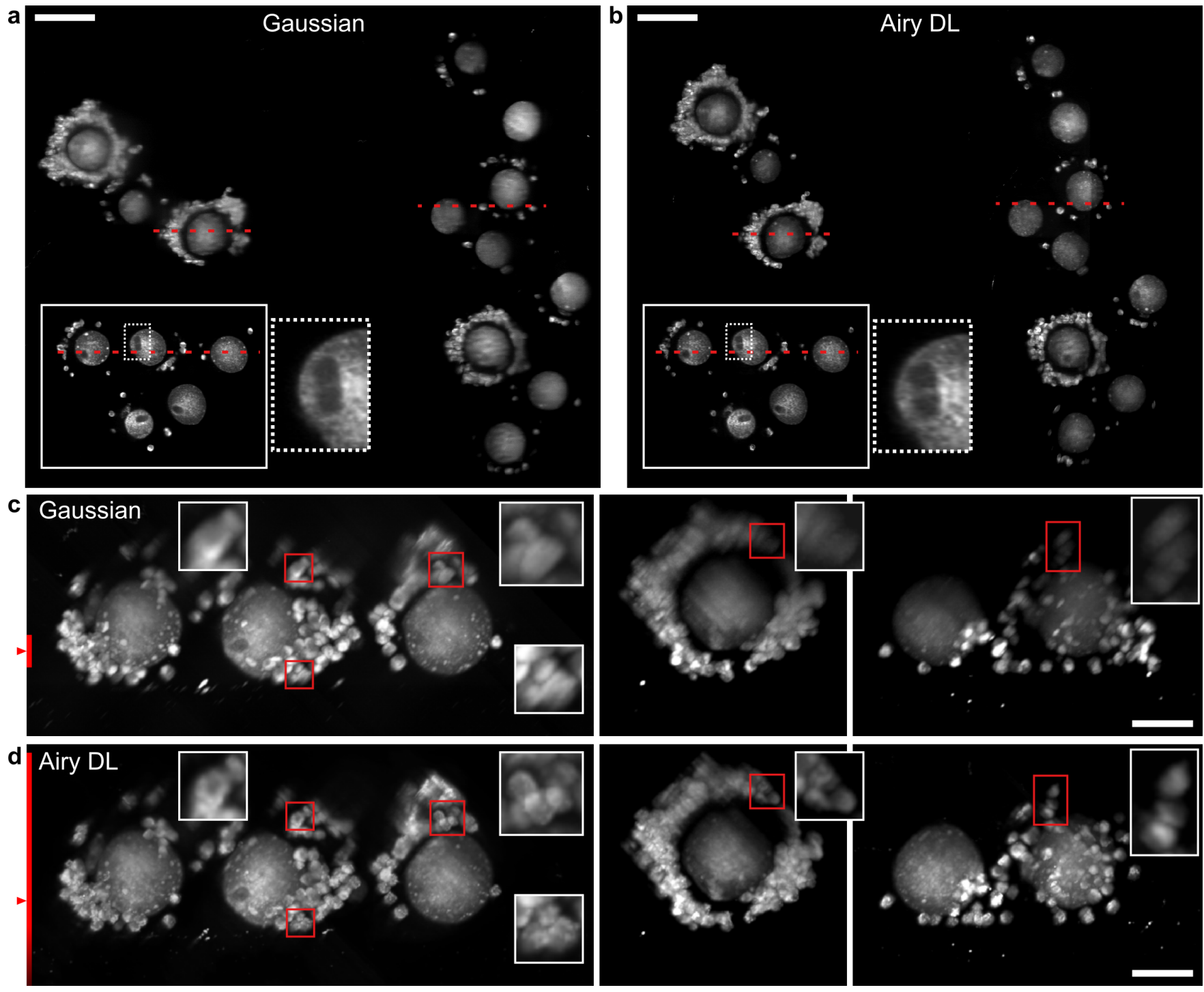

Fig. 5. Widefield LSM images of mouse cumulus oocyte complexes (COCs) with a Gaussian (a,c) and Airy beams with deep learning deconvolution (b,d). (a,b) Enface sections of the Gaussian (a) and Airy (b) LSM images. The inset marked by a solid line in (a,b) is taken from another sample using identical imaging parameters. Scale bars in $(\mathrm{a}, \mathrm{b})$ are $100 \mu \mathrm{m}$. The zoomed-in region marked by a dashed line depicts the barrel-shaped spindle and metaphase plate of meiosis II. The red dashed lines mark the location of the cross-sections in (c,d). (c,d) Cross-sectional maximum intensity projections (range: $50 \mu \mathrm{m}$ ). The insets correspond to regions marked by red rectangles. The red line on the left of (c,d) shows the theoretical DOF of the Gaussian and Airy beams. The red triangle marks the focal position. Scale bars in (c,d) are $50 \mu \mathrm{m}$.

specting the areas marked by the red asterisk, matches that seen in the blastocysts (Fig. 4). Image quality in the Gaussian LSM rapidly deteriorates with depth, showing substantial blurring and loss of contrast. However, image quality is preserved in depth with Airy DL LSM, distinguishing sharper fluorescent features. This is particularly evident at $52 \mu \mathrm{m}$ depth in the regions marked by the red triangles. The capacity to image optically thick sections of the brain with high resolution and contrast enables the inspection of axonal connections with minimal damage, in contrast with, for instance, microtome sectioning needed for conventional fluorescence microscopy. Airy DL LSM provides attractive opportunities for detailed studies of morphology, neurodegeneration and broader pathogenesis in neuroscience.

\section{Discussion}

We have demonstrated that learned deconvolution of structured LSM, in particular, using the Airy light field, can decode image content with a high contrast, over an extended field of view and additionally provide super-resolution capacity. Specifically, we show that deep learning is superior to conventional deconvolution in its ability to achieve a more symmetric deconvolved PSF, a 98\% SSIM vs. 85-91\% for RL deconvolution, and a 3-fold improvement in the tolerance to a mismatched PSF. Importantly, we also show that the combined Airy DL method exceeds the performance of a conventional Gaussian LSM, exceeding both the resolution (by a factor of $\sqrt{2}$ ) and the contrast, as illustrated by the MTF (Fig. 4(f)). It is important to note that the use of propagationinvariant beams leads to a reduction in contrast due to the distribution of power into the side lobes (8), establishing a trade-off in resolution, contrast and DOF. Airy DL extends on 

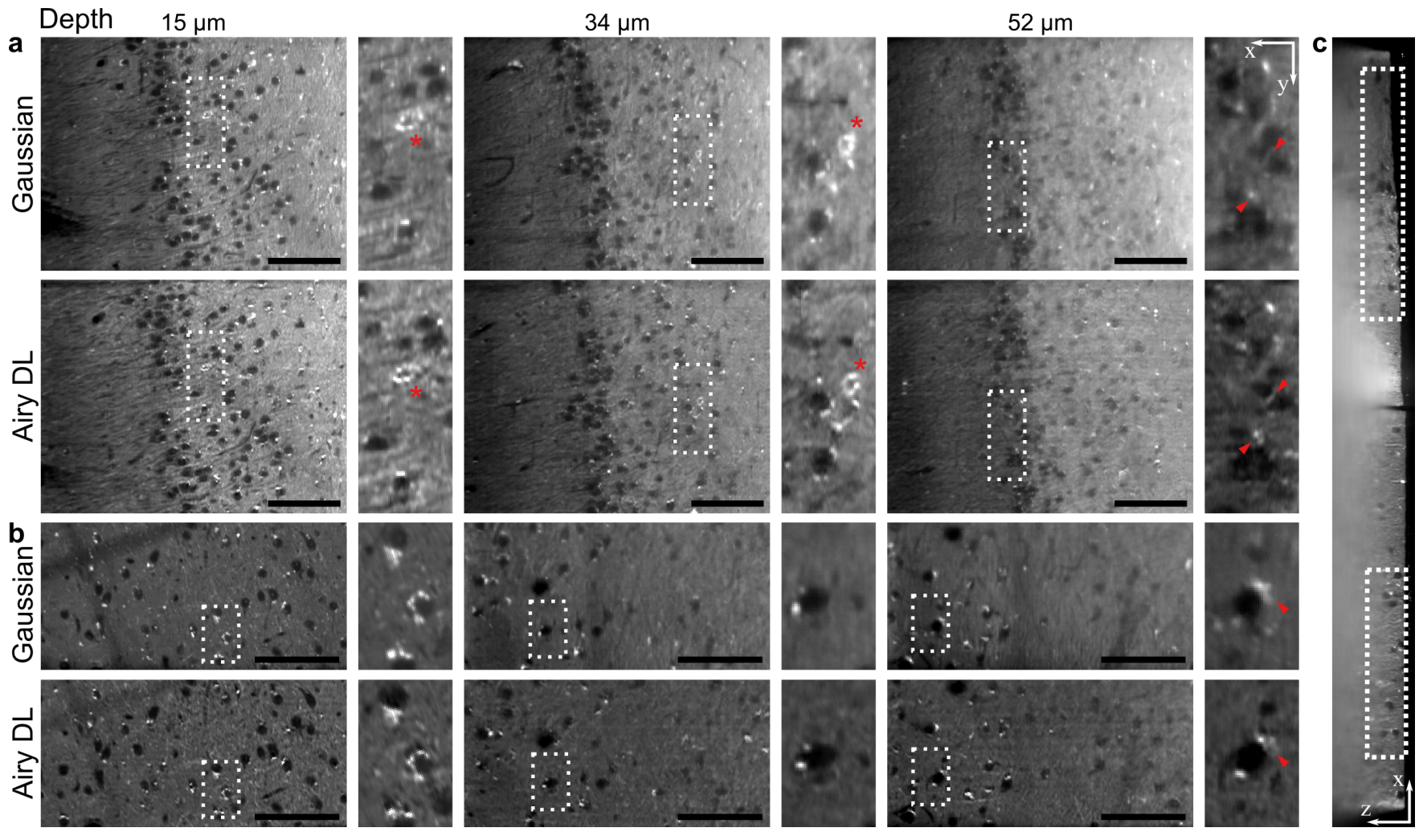

Fig. 6. Excised brain tissue from a mouse expressing enhanced YFP in parvalbumin-positive (PV+) neurons. (a, b) Enface sections in depth imaged using a Gaussian and Airy LSM with deep learning deconvolution. Sections correspond to the (a) hippocampus and (b) deeper cortical regions. Insets to the right correspond to the regions marked by a dashed rectangle. Red asterisks mark PV+ neurons. Red triangles mark regions that illustrate enhanced performance from the combination of an Airy beam and deep learning at depth. (c) Cross-sectional image ( $z$ is depth) showing the regions displayed in (a) and (b). Scale bars are $100 \mu \mathrm{m}$.

all these parameters at once, making it an attractive method over a conventional Gaussian LSM, and offers new opportunities for rapid multiscale bioimaging, such as in embryology and neuroscience.

Such performance is rarely afforded by conventional numerical solutions to the inverse problem of imaging. This is because many algorithms are designed to optimise the solution with little consideration of image, content or physics priors. For instance, in the case of RL deconvolution, a maximum-likelihood solution is reached for a general case. DL, however, by its nature (35) and careful design, takes advantage of learned priors to preform more challenging underdetermined inversions (15). Specifically to this work, we leverage sparsity that is present in natural images and the estimate of the forward operator of LSM imaging to exclude those solutions that cannot be practical in our imaging configuration. We note that priors can be utilised in conventional deconvolution algorithms, especially in those seeking superresolution (3). However, complexity in these methods is often met with longer computation time, and ill-posed nature often requires high signal-to-noise in the experiment, for instance, in SRRF (44) which can require more than 300 images in one location. DL provides an accessible means to deconvolution with learned priors with rapid feed-forward inference (here, $0.2 \mathrm{~s}$ per widefield image). Its capacity to learn non-linear functions enables inversions at exceptionally low signals, including from single photon fluences $(16,20)$. Further, the support of DL by contemporary hardware, such as the GPU, promise ultrafast and even real-time processing with a low barrier-to-entry.

Beyond addressing structured PSFs, a key property and advantage of our network is that it is experimentally unsupervised. Learned priors combined with perceptual consistency of experimental images is sufficient to achieve deconvolution and super-resolution. This simplifies the requirements to solely images that would be collected in a standard experiment. Further, this achieves generality, which we demonstrate by utilising the same trained network for all deconvolutions of a single beam shape, regardless of sample. This physics-informed learning is a marked contrast to data-driven approaches $(17,18)$, which derive their performance, in part, from sample-specific priors, thus, requiring training for each class of sample. The use of physics theory to train neural networks has emerged in recent works (15), including in correcting for scattering (45) and improving resolution (24) in microscopy.

We have demonstrated learned deconvolution experimentally using the Airy light field. However, we expect similar improvements when using other structured beams, such as the Bessel beam, due their shared propagation-invariance and self-healing properties, and from our own simulations (Fig. 2). Bessel beams are challenging to form by an SLM in the pupil plane due to their thin ring profile (46). However, Airy and Bessel beams can be generated readily using cheap optical components $(8,13,14)$. The combination of DL with compact and inexpensive LSM may provide a powerful and 
accessible tool for bioimaging. Further, aberrations from alignment may be incorporated in the network via Zernike polynomials (23). Combined with increased tolerance to model mismatch (Fig. 2(c)), the impact of misalignment may be minimised with DL. This may further democratise other precision LSM setups, such as the lattice light sheet (12) and more broadly other microscopic approaches that employ deconvolution.

\section{Conclusion}

We have demonstrated learned deconvolution of structured PSFs in LSM, leading to the recovery of high-resolution and high-contrast information over extended fields of view. Our method is experimentally unsupervised and has demonstrated good performance and generality. The application to embryology and neuroscience show attractive potential across a range of bioimaging studies. Our method, which is provided to the reader as open source, may enable ready implementation accessible to many imaging setups.

\section{Materials and Methods}

Network. Let us consider the LSM imaging process described by $y=H(x)+\epsilon$, where $x$ is the ground truth (GT), $y$ is the recorded image (LR), $H$ is the imaging operator that we seek to invert, and $\epsilon$ is some noise. The goal of deep learning is to train a network, $G$, such that is provides a good estimate of the ground truth (DL) from LR images, $\hat{x}=G(y)$. This is achieved by minimising a training loss function (TLF), $\mathcal{L}_{G}$, i.e., $\operatorname{argmin}_{G}\left\{\mathcal{L}_{G}\right\}$. We implement this process using a generative adversarial network (GAN) (26), where $G$ is trained concurrently with another adversarial discriminator network, $D$, that seeks to maximise its ability to discriminate between $x$ and $\hat{x}$. The performance of $D$ features in $\mathcal{L}_{G}$ and leads $G$ towards optimising the adversarial min-max game (26).

The discriminator, $D$, is trained to minimise the following TLF based on least-squares (47):

$$
\mathcal{L}_{D}=\frac{1}{2} \mathbb{E}\left[\left(D(x)-\delta_{T}\right)^{2}\right]+\frac{1}{2} \mathbb{E}\left[\left(D(G(y))-\delta_{F}\right)^{2}\right],
$$

where $\delta_{T}$ and $\delta_{F}$ are the 'true' and 'false' labels, and $\mathbb{E}$ is the expected value evaluated over the entire training batch. We utilise one sided smooth labels, i.e., $\delta_{T}=0.9$ and $\delta_{F}=0$ to prevent $D$ from over-saturating learning from a particular strong feature. $D$ is based on the Patch GAN architecture (29) (detailed in Supplementary Note 2).

The Generator, $G$, is trained to minimise a linear combination of three TLFs:

$$
\mathcal{L}_{G}=\mathcal{L}_{A d v}+\lambda \mathcal{L}_{L 1}+\gamma \mathcal{L}_{\text {Per }}
$$

which are the adversarial, $L_{1}$-norm and perceptual losses, respectively. Weights $\lambda$ and $\gamma$ are chosen such that $\mathcal{L}_{A d v}$ and $\mathcal{L}_{L 1}$ have approximately equal contribution to $\mathcal{L}_{G}$ at the end of training (here, $\lambda=30$ ). Perceptual loss acts as a regulariser, and was manually chosen as $1 \times 10^{-4}$. $G$ is based on a 16-layer residual network (ResNet) (28), inspired by SRGAN (27) (detailed in Supplementary Note 2).
We use multi-objective training (48), i.e., we train two independent discriminators, $D_{1}$ and $D_{2}$. Using each discriminator, we evaluate a least-squares adversarial loss (47):

$$
\mathcal{L}_{A d v_{1,2}}=\mathbb{E}\left[\left(D(G(y))-\delta_{T}\right)^{2}\right] .
$$

The minimum of the two is used to update the gradients of $G$ :

$$
\mathcal{L}_{A d v}=\min \left\{\mathcal{L}_{A d v_{1}}, \mathcal{L}_{A d v_{2}}\right\} .
$$

Multi-objective training prevents a singe $D$ and $G$ being locked into a min-max game over a single strong feature. In such a case, the second discriminator becomes momentarily free of the min-max game, and is able to reestablish a good training gradient.

Pixel loss using $L_{1}$-norm establishes spatial saliency between DL $(G(y))$ and GT $(x)$ images, and is given as:

$$
\mathcal{L}_{L 1}=\mathbb{E}\left[\|G(y)-x\|_{1}\right] .
$$

Perceptual loss is provided with a pre-trained and publicly available VGG-16 (30) network, $V . V$ is tuned towards the classification of image content. Inference of an image leads to its layers possessing some quantification of image content that is less dependent on its precise pixel values (31). Specifically, we compare LR and DL image content by extracting intermediate features of their $V$ inference:

$$
\mathcal{L}_{P e r}=\mathbb{E}\left[\sum_{i}\left(V^{i}\left(G\left(y^{\prime}\right)\right)-V^{i}\left(y^{\prime}\right)\right)^{2}\right],
$$

where $i$ are the selected layers of $V$.

It is important to note that $\mathcal{L}_{A d v}$ and $\mathcal{L}_{L 1}$ are calculated using the DL and GT images that are simulated based on the known light propagation in the LSM (Fig. 1). Real acquired LR images feature solely in $\mathcal{L}_{P e r}$. Perceptual loss, in this instance, plays an important role in directing $G$ towards good performance in real tissues. If a pixel-wise loss was to be used instead, it would act to make DL images look like LR images, which is counter-productive. Instead, perceptual loss provides two important features. First, it focuses on preserving content that is present in the LR images, namely, the distribution of spectral density, contrast and brightness, and the low-resolution content that should be unaffected by the deconvolution process. Second, it flags network artefacts, such as fixed pattern noise, with strong increases in loss. This makes it a valuable regularisation parameter to bridge mismatch between the content that can be efficiently simulated and real samples. Perceptual loss has previously been valuable for phase-retrieval in microscopy (16).

Training and Inference. Training was performed using simulated LR images and their GT, and real LR images of tissue. All inputs and DL outputs were $64 \times 64$ pixel grayscale images. LR images were simulated by convolving a sparse or speckle bases (Fig. 1(b)) with a known PSF. The PSF was evaluated by superimposing a Gaussian detection beam orthogonal to an illumination beam. The beam propagation models are described in the subsequent sections. GT images 
were simulated by convolving the same basis used for LR with a Gaussian spot at $1 / \sqrt{2}$ the diffraction limit. Real LR images were retrieved from an LSM volume of a blastocyst in an automated fashion by identifying the brightest region of interest in each cross-section and applying a random spatial shift. Simulated LR and GT images were normalised to a range of $(0.1,0.9)$ to avoid clipping. The real LR images were scaled to the minimum and maximum intensity of the entire LSM volume.

Training for each beam type was performed using a total of 512 sparse and 512 speckle simulations, and 215 LR images from a blastocyst volume. Of there a random selection of 32 sparse and 32 speckle LR images were used for validation. Due to the adversarial nature of GAN training, no early stopping was used. This is because adversarial loss is not an indication of convergence. We used a batch size of 8 and trained for 300 epochs. Gradient-based optimisation was performed using the Adam optimiser with learning rates of $1 \times 10^{-4}$ and $4 \times 10^{-4}$ for $G$ and $D$, respectively, and hyper-parameters $\beta_{1}=0.5$ and $\beta_{2}=0.999$. Training was performed using PyTorch and an NVidia RTX 2060, and took approximately 2.5 hours. Inference on widefield images was performed by a $64 \times 64$ sliding window with an 8 -pixel overlap to avoid boundary issues. Inference took $3 \mathrm{~ms}$ for a single LR image, which corresponds to approximately $0.5 \mathrm{~s}$ for a widefield LSM image. We replicated training and inference on an NVidia RTX 3090, which reduced the total training time to 1.2 hours, and inference time to $1 \mathrm{~ms}$ and $0.2 \mathrm{~s}$ for single and widefield sections, respectively.

Imaging system. The imaging setup is illustrated in Figure 1(a). A 488-nm laser source (TOPTICA, Germany) was used for illumination. The beam was expanded with an objective (Obj ${ }_{\mathrm{B}}$, RMS10X, 0.25NA, 10X, Olympus) and lens (L1, $\mathrm{f}: 150 \mathrm{~mm}$ ), and apodised using an aperture. The beam was filtered spectrally using a notch filter $\left(\lambda_{c}: 480 \mathrm{~nm}, \Delta \lambda: 17 \mathrm{~nm}\right)$ and spatially using a pinhole $(15-\mu \mathrm{m}$ diameter $)$ at the objective focus. A half wave plate controlled polarisation to maximise the efficiency of the spatial light modulator (SLM, X10468, Hamamatsu, Japan). Phase and amplitude was controlled by using the SLM in diffraction mode. The desired phase was projected onto the SLM and the desired intensity was modulated by a phase ramp, realising a blazed grating. The first-order diffraction was spatially filtered using an aperture (SF). The beam was relayed by lenses (L2-L5, f:250, $100,50,75 \mathrm{~mm})$ and the illumination objective $\left(\mathrm{Obj}_{\mathrm{I}}, 54-10-\right.$ 12, 0.367NA 4X, Navitar). The light sheet was generated by a galvo scanner (Galvo, Thorlabs, NJ) in the Fourier plane. Fluorescence was collected by the detection objective $\left(\mathrm{Obj}_{\mathrm{D}}\right.$, 54-10-12, 0.367NA 4X, Navitar) and relayed to the camera (CAM, Iris 15, Teledyne Photometrics, AZ) by a tube lens (TL, f:200 mm). Fluorescence was filtered using a bandpass filter (BP, $\lambda_{c}: 532 \mathrm{~nm}, \Delta \lambda: 50 \mathrm{~nm}$ ) and a notch filter (NF, $\left.\lambda_{c}: 488 \mathrm{~nm}\right)$.

Acquisition. LSM volumes were acquired by placing the sample at the focus of the objectives and laterally scanning using a motorised transducer (M-230.10, Physik Instrumente
Ltd). Scanning position was synchronised to the camera acquisition, and images were acquired in $0.5-\mu \mathrm{m}$ steps. Due to the geometry illustrated in the inset in Figure 1(a), lateral scanning was at $45^{\circ}$ to the detection plane (also detailed in (41)). Due to this shearing, acquired image stacks were transformed to the physical Cartesian coordinate space using in-house built software (MATLAB, MA) based on interpolation. All LSM images presented in this paper were sampled with an isotropic $0.85 \mu \mathrm{m}$ pixel size.

Structured light fields. The description of the beams used in this work is provided in Supplementary Note 3. Briefly, all beam shapes are described in the pupil plane. The Gaussian beam is described by its $1 / e^{2}$ waist size $\left(w_{0}\right)$. The Airy beam is described by the $1 / e^{2}$ waist size $\left(w_{0}\right)$ of a Gaussian intensity and a scale-invariant $\alpha$ parameter that represents the cubic phase modulation rate. The Bessel beam is described by a ring with a $1 / e^{2}$ waist size $\left(w_{0}\right)$ and a ring radius $(r r)$. Table 1 lists the beam parameters used in this work.

$\begin{array}{llll}\text { Beam } & \text { Parameters }(\mathbf{m m}) & \text { Res. }(\mu \mathbf{m}) & \text { DOF }(\mu \mathbf{m}) \\ \text { Gaussian 1 (G1) } & w_{0}: 1.02 & 2.5 & 27 \\ \text { Gaussian 0.5 (G0.5) } & w_{0}: 0.510 & 5.3 & 107 \\ \text { Airy 0.5 (A0.5) } & w_{0}: 1.38 ; \alpha: 0.5 & 2.6 & 169 \\ \text { Airy 1 (A1) } & w_{0}: 1.53 ; \alpha: 1 & 2.5 & 261 \\ \text { Airy 2 (A2) } & w_{0}: 1.73 ; \alpha: 2 & 2.5 & 372 \\ \text { Bessel 0.8 (B0.8) } & w_{0}: 0.04 ; r r: 0.8 & 3.7 & 510 \\ \text { Bessel 1.1 (B1.1) } & w_{0}: 0.055 ; r r: 1.1 & 2.8 & 276\end{array}$

Table 1. Illumination beam properties. Res: axial resolution. DOF: depth of focus.

Phantoms. Phantoms were utilised to characterise the system's PSF and demonstrate DL performance. Phantoms were fabricated from 200-nm diameter green fluorescent microspheres (G200, Duke Scientific, CA) manually mixed with $1.5 \%$ agarose. The samples were pipetted into and cured in a 3D-printed sample holder. The sample holder featured a thin fluorinated ethylene propylene (FEP) window for imaging and index matching (RI: 1.344).

Mouse oocytes and embryos. Female (21-23 days) CBA $x$ C57BL/6 first filial (CBAF1) generation mice were obtained from Laboratory Animal Services (University of Adelaide, Australia) and maintained on a $12 \mathrm{~h}$ light: $12 \mathrm{~h}$ dark cycle with rodent chow and water provided ad libitum. All studies were approved by the University of Adelaide's Animal Ethics Committee and were conducted in accordance with the Australian Code of Practice for the Care and Use of Animals for Scientific Purposes.

Female mice were administered intraperitoneally (i.p.) with $5 \mathrm{IU}$ of equine chorionic gonadotropin (eCG; Folligon, Braeside, VIC, Australia), followed by 5 IU human chorionic gonadotrophin (hCG, i.p.; Kilsyth, VIC, Australia) 48 $\mathrm{h}$ later. Mice were culled by cervical dislocation $14 \mathrm{~h}$ posthCG administration and the oviducts carefully removed. $\mathrm{Cu}-$ mulus oocyte complexes (COCs) were harvested by gently puncturing the ampullae of the oviduct using a 29-gauge insulin syringe with needle (Terumo Australia Pty Ltd, Australia). These COCs were either fixed immediately in $4 \%$ 
bioRxiv preprint doi: https://doi.org/10.1101/2021.05.26.445797; this version posted October 15, 2021. The copyright holder for this preprint (which was not certified by peer review) is the author/funder, who has granted bioRxiv a license to display the preprint in perpetuity. It is made available under aCC-BY 4.0 International license.

paraformaldehye (PFA) or co-incubated with sperm for IVF. In vitro fertilisation (IVF) occurred through co-incubation of matured COCs with sperm in Research Fertilisation medium (ARTLab Solutions, Australia) for $4 \mathrm{~h}$ at $37^{\circ} \mathrm{C}$. Resulting presumptive zygotes were transferred into Research Cleave medium (ARTLab Solutions, Australia) under paraffin oil and allowed to develop to the blastocyst-stage (96 h postIVF) at $37^{\circ} \mathrm{C}$ in $5 \% \mathrm{O}_{2}$.

Cumulus oocyte complexes (COCs) and blastocyst-stage embryos were fixed in 4\% PFA for 30 mins at room temperature, followed by washes in PBV (phosphate buffer saline; PBS containing $0.3 \mathrm{mg} / \mathrm{ml}$ of polyvinyl alcohol; PVA). Fixed COCs and blastocyst-stage embryos were mounted under oil on a 3D-printed sample holder with an FEP window for imaging.

Mouse brain tissue. All animal experiments in Figure 6 were performed in accordance with the United Kingdom Animals (Scientific Procedures) Act of 1986 Home Office regulates and approved by the Home Office (PPL70/8883). Detailed procedures are described elsewhere (49). Briefly, a female adult (8.4 months old) PV-Cre::Ai32 mouse (PV-Cre, JAX008069; Ai32, JAX012569) was used. This mouse expressed channelrhodopsin-2 (ChR2) tagged with enhanced YFP (EYFP) in parvalbumin-positive $(\mathrm{PV}+)$ neurons. The mouse was deeply anaesthetised with a mixture of pentobarbital and lidocaine, and perfused transcardially with physiological saline followed by $4 \%$ paraformaldehyde $/ 0.1 \mathrm{M}$ phosphate buffer, $\mathrm{pH}$ 7.4. After an overnight post-fixation in the same fixative, the brain was immersed in $30 \%$ sucrose in phosphate buffered saline (PBS) at $4^{\circ} \mathrm{C}$ for cryoprotection. The brain was cut into 1-mm thick coronal sections and kept in PBS until imaged. The imaging was focused on the cortex and hippocampus regions.

Acknowledgements. We would like to acknowledge Federico Gasparoli for early support in constructing the imaging system and Mirna Merkler for preparing the excised mouse brain section. We acknowledge Erik Linder-Norén's implementations of GANs in PyTorch (https://github. com/eriklindernoren/PyTorch-GAN) that instantiated the code developed in this project.

Data Availability. Code used to simulate light-sheet microscopy and perform deep learning is available publicly at https://github.com/philipwijesinghe/ learned-deconvolution. All data underpinning this study is available at https://doi.org/10.17630/ bf92bc18-0b81-41f7-bd44-d74040af7cf0.

Funding. This project was funded by the UK Engineering and Physical Sciences Research Council (grants EP/P030017/1 and EP/R004854/1), and has received funding from the European Union's Horizon 2020 research and innovation programme under grant agreement (EC-GA 871212) and H2020 FETOPEN project "Dynamic" (EC-GA 863203). KRD is supported by a Mid-Career Fellowship from the
Hospital Research Foundation (C-MCF-58-2019). SS was funded by BBSRC (BB/M00905X/1).

Author Contribution. PW and KD initiated the project. PW designed the network and imaging theory. SC built the setup and performed imaging. PW performed image processing, network training and image analysis. DJXC and KRD prepared the embryo samples, and provided training and embryo image analysis. SS provided brain tissue, training and analysed brain data. PW wrote the manuscript with input from all authors. All authors reviewed and approved the work. KD supervised the project.

Conflicts of Interest. The authors declare no conflicts of interest.

\section{Bibliography}

1. Sibarita, J.-B. Deconvolution Microscopy. In Rietdorf, J. (ed.) Microscopy Techniques: --, Advances in Biochemical Engineering, 201-243 (Springer, Berlin, Heidelberg, 2005). URL https://doi.org/10.1007/b102215.

2. McNally, J. G., Karpova, T., Cooper, J. \& Conchello, J. A. Three-Dimensional Imaging by Deconvolution Microscopy. Methods 19, 373-385 (1999). URL https://www.sciencedirect.com/science/article/pii/ S1046202399908733.

3. Sage, D. et al. DeconvolutionLab2: An open-source software for deconvolution microscopy. Methods 115, 28-41 (2017). URL https://www.sciencedirect.com/science/article/pii/ S1046202316305096.

4. Barbastathis, G., Ozcan, A. \& Situ, G. On the use of deep learning for computational imaging. Optica 6, 921-943 (2019). URL https://www.osapublishing.org/optica/abstract.cfm? uri=optica-6-8-921.

5. Gustafsson, M. G. L. Nonlinear structured-illumination microscopy: Wide-field fluorescence imaging with theoretically unlimited resolution. Proceedings of the National Academy of Sciences 102, 13081-13086 (2005). URL https: //www. pnas.org/content/102/37/13081.

6. Neil, M. a. A., Juškaitis, R. \& Wilson, T. Method of obtaining optical sectioning by using structured light in a conventional microscope. Optics Letters 22, 1905-1907 (1997). URL https://www.osapublishing.org/ol/ abstract. cfm?uri=ol-22-24-1905.

7. Shechtman, Y., Weiss, L. E., Backer, A. S., Lee, M. Y. \& Moerner, W. E. Multicolour localization microscopy by point-spread-function engineering. $\mathrm{Na}$ ture Photonics 10, 590-594 (2016). URL https://www. nature.com/ articles/nphoton.2016.137.

8. Vettenburg, T. et al. Light-sheet microscopy using an Airy beam. Nature Methods 11, 541-544 (2014). URL http://www. nature.com/nmeth/ journal/v11/n5/abs/nmeth.2922.html.

9. Planchon, T. A. et al. Rapid three-dimensional isotropic imaging of living cells using Bessel beam plane illumination. Nature Methods 8, 417-423 (2011). URL https: / / www . nature.com/articles/nmeth.1586.

10. Nylk, J., McCluskey, K., Aggarwal, S., Tello, J. A. \& Dholakia, K. Enhancement of image quality and imaging depth with Airy light-sheet microscopy in cleared and non-cleared neural tissue. Biomedical Optics Express 7, 40214033 (2016). URL https://www. osapublishing.org/abstract. cfm?uri=boe-7-10-4021.

11. Fahrbach, F. O., Simon, P. \& Rohrbach, A. Microscopy with self-reconstructing beams. Nature Photonics 4, 780-785 (2010). URL https://www. nature.com/articles/nphoton.2010.204.

12. Chen, B.-C. et al. Lattice light-sheet microscopy: Imaging molecules to embryos at high spatiotemporal resolution. Science 346, 1257998 (2014). URL http://science.sciencemag.org/content/346/ $6208 / 1257998$.

13. Yang, Z. et al. A compact Airy beam light sheet microscope with a tilted cylindrical lens. Biomedical Optics Express 5, 3434-3442 (2014). URL https : / / www. osapublishing.org/abstract. cfm?uri=boe-5-10-3434.

14. Arlt, J. \& Dholakia, K. Generation of high-order Bessel beams by use of an axicon. Optics Communications 177, 297-301 (2000). URL https://www.sciencedirect.com/science/article/pii/ So030401800005721.

15. Wijesinghe, P. \& Dholakia, K. Emergent physics-informed design of deep learning for microscopy. Journal of Physics: Photonics (2021). URL http: //iopscience.iop.org/article/10.1088/2515-7647/abf02c.

16. Deng, M., Goy, A., Li, S., Arthur, K. \& Barbastathis, G. Low Photon Budget Phase Retrieval with Perceptual Loss Trained Deep Neural Networks. arXiv:1906.05687 [eess] (2019). URL http://arxiv.org/abs/1906. 05687. 
bioRxiv preprint doi: https://doi.org/10.1101/2021.05.26.445797; this version posted October 15, 2021. The copyright holder for this preprint (which was not certified by peer review) is the author/funder, who has granted bioRxiv a license to display the preprint in perpetuity. It is made available under aCC-BY 4.0 International license.

17. Weigert, M. et al. Content-aware image restoration: pushing the limits of fluorescence microscopy. Nature Methods 15, 1090-1097 (2018). URL https://www. nature.com/articles/s41592-018-0216-7.

18. Wang, H. et al. Deep learning enables cross-modality super-resolution in fluorescence microscopy. Nature Methods 16, 103 (2019). URL https: //www.nature.com/articles/s41592-018-0239-0.

19. Belthangady, C. \& Royer, L. A. Applications, promises, and pitfalls of deep learning for fluorescence image reconstruction. Nature Methods 16, 1215-1225 (2019). URL https://www. nature.com/articles/ s41592-019-0458-z.

20. Goy, A., Arthur, K., Li, S. \& Barbastathis, G. Low Photon Count Phase Retrieval Using Deep Learning. Physical Review Letters 121, 243902 (2018). URL https://link.aps.org/doi/10.1103/PhysRevLett. 121.243902 .

21. Deng, M., Li, S., Goy, A., Kang, I. \& Barbastathis, G. Learning to synthesize: robust phase retrieval at low photon counts. Light: Science \& Applications 9, 36 (2020). URL https://www.nature.com/articles/ S41377-020-0267-2.

22. Wang, F. et al. Phase imaging with an untrained neural network. Light: Science \& Applications 9, 77 (2020). URL https: / / www . nature.com/ articles/s41377-020-0302-3.

23. Bostan, E., Heckel, R., Chen, M., Kellman, M. \& Waller, L. Deep phase decoder: self-calibrating phase microscopy with an untrained deep neural network. Optica 7, 559-562 (2020). URL https: //www. osapublishing. org/optica/abstract. cfm?uri=optica-7-6-559.

24. Zhang, $\mathrm{H}$. et al. High-throughput, high-resolution deep learning microscopy based on registration-free generative adversarial network. Biomedical Optics Express 10, 1044-1063 (2019). URL https: //www. osapublishing. org/boe/abstract. cfm?uri=boe-10-3-1044.

25. Li, S., Deng, M., Lee, J., Sinha, A. \& Barbastathis, G. Imaging through glass diffusers using densely connected convolutional networks. Optica 5, 803-813 (2018). URL https://www.osapublishing.org/optica/ abstract. cfm?uri=optica-5-7-803.

26. Goodfellow, I. et al. Generative Adversarial Nets. In Ghahramani, Z., Welling, M., Cortes, C., Lawrence, N. D. \& Weinberger, K. Q. (eds.) Advances in Neural Information Processing Systems 27, 2672-2680 (Curran Associates, Inc., 2014). URL http://papers.nips.cc/paper/ 5423-generative-adversarial-nets.pdf.

27. Ledig, C. et al. Photo-Realistic Single Image Super-Resolution Using a Generative Adversarial Network. arXiv:1609.04802 [cs, stat] (2017). URL http://arxiv.org/abs/1609.04802.

28. He, K., Zhang, X., Ren, S. \& Sun, J. Deep Residual Learning for Image Recognition. In Proceedings of the IEEE Conference on Computer Vision and Pattern Recognition, 770-778 (2016). URL https://openaccess.thecvf.com/content_cvpr_2016/html/ He_Deep_Residual_Learning_CVPR_2016_paper.html.

29. Isola, P., Zhu, J.-Y., Zhou, T. \& Efros, A. A. Image-to-Image Translation with Conditional Adversarial Networks. In 2017 IEEE Conference on Computer Vision and Pattern Recognition (CVPR), 5967-5976 (2017).

30. Simonyan, K. \& Zisserman, A. Very Deep Convolutional Networks for LargeScale Image Recognition. arXiv:1409.1556 [cs] (2015). URL http:// arxiv.org/abs/1409.1556.

31. Johnson, J., Alahi, A. \& Fei-Fei, L. Perceptual Losses for Real-Time Style Transfer and Super-Resolution. In Leibe, B., Matas, J., Sebe, N. \& Welling, M. (eds.) Computer Vision - ECCV 2016, Lecture Notes in Computer Science, 694-711 (Springer International Publishing, Cham, 2016).

32. Li, S. \& Barbastathis, G. Spectral pre-modulation of training examples enhances the spatial resolution of the phase extraction neural network (PhENN). Optics Express 26, 29340-29352 (2018). URL https: //www . osapublishing.org/oe/abstract. cfm?uri=oe-26-22-29340.

33. Richardson, W. H. Bayesian-Based Iterative Method of Image Restoration*. JOSA 62, 55-59 (1972). URL https://www.osapublishing.org/ josa/abstract.cfm?uri=josa-62-1-55.

34. Lucy, L. B. An iterative technique for the rectification of observed distributions. The Astronomical Journal 79, 745 (1974). URL http://adsabs. harvard.edu/abs/1974AJ....79.745L.

35. Ulyanov, D., Vedaldi, A. \& Lempitsky, V. Deep Image Prior. International Journal of Computer Vision 128, 1867-1888 (2020). URL https://doi. org/10.1007/s11263-020-01303-4.

36. Huisken, J. \& Stainier, D. Y. R. Selective plane illumination microscopy techniques in developmental biology. Development 136, 1963-1975 (2009). URL http://dev.biologists.org/content/136/12/1963.

37. Gosnell, M. E. et al. Quantitative non-invasive cell characterisation and discrimination based on multispectral autofluorescence features. Scientific $R e-$ ports 6, 23453 (2016). URL https://www.nature.com/articles/ srep23453.

38. Xenopoulos, P., Kang, M. \& Hadjantonakis, A.-K. Cell lineage allocation within the inner cell mass of the mouse blastocyst. Results and Problems in Cell Differentiation 55, 185-202 (2012).

39. Richani, D., Dunning, K. R., Thompson, J. G. \& Gilchrist, R. B. Metabolic co-dependence of the oocyte and cumulus cells: essential role in determining oocyte developmental competence. Human Reproduction Update 27, 27-47 (2021). URL https://doi.org/10.1093/humupd/dmaa043.

40. Corsetti, S., Gunn-Moore, F. \& Dholakia, K. Light sheet fluorescence microscopy for neuroscience. Journal of Neuroscience Methods (2018). URL http://www. sciencedirect. com/science/article/ pii/s0165027018302188.

41. Corsetti, S. et al. Widefield light sheet microscopy using an Airy beam combined with deep-learning super-resolution. OSA Continuum 3, 1068-1083 (2020). URL https://www.osapublishing.org/osac/abstract. cfm?uri=osac-3-4-1068.

42. Freund, T. F. \& Buzsáki, G. Interneurons of the hippocampus. Hippocampus 6, 347-470 (1996). URL https://onlinelibrary.wiley.com/doi/

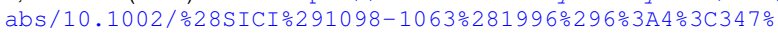
$3 \mathrm{~A} \div 3 \mathrm{AATD}-\mathrm{HIPO} \div 3 \mathrm{E} 3 \cdot 0 . \mathrm{CO} \div 3 \mathrm{~B} 2-\mathrm{I}$.

43. Kawaguchi, Y. \& Kubota, Y. GABAergic cell subtypes and their synaptic connections in rat frontal cortex. Cerebral Cortex 7, 476-486 (1997). URL https://doi.org/10.1093/cercor/7.6.476.

44. Gustafsson, N. et al. Fast live-cell conventional fluorophore nanoscopy with ImageJ through super-resolution radial fluctuations. Nature Communications 7, 12471 (2016). URL https://www.nature.com/articles/ ncomms 12471.

45. Xiao, L. et al. Network-enabled efficient image restoration for $3 \mathrm{D} \mathrm{mi}-$ croscopy of turbid biological specimens. bioRxiv 2020.05.27.118869 (2020). URL https://www.biorxiv.org/content/10.1101/2020. $05.27 .118869 \mathrm{v} 1$.

46. Čižmár, T. et al. Generation of multiple Bessel beams for a biophotonics workstation. Optics Express 16, 14024-14035 (2008). URL https : //www . osapublishing.org/oe/abstract. cfm?uri=oe-16-18-14024.

47. Mao, $X$. et al. Least Squares Generative Adversarial Networks. arXiv:1611.04076 [cs] (2017). URL http://arxiv.org/abs/1611. 04076.

48. Albuquerque, I. et al. Multi-objective training of Generative Adversarial Networks with multiple discriminators. arXiv:1901.08680 [cs, stat] (2019). URL http://arxiv.org/abs/1901.08680.

49. Lyngholm, D. \& Sakata, S. Cre-Dependent Optogenetic Transgenic Mice Without Early Age-Related Hearing Loss. Frontiers in Aging Neuroscience 11 (2019). URL https://www. frontiersin.org/articles/10.3389/ fnagi.2019.00029/full. 